\title{
A Novel Reliability Enhanced Handoff Method in Future Wireless Heterogeneous Networks
}

\author{
YuPeng Wang , and KaiLi Li \\ Shenyang Aerospace University, Department of Electrical and Information Engineering, Shenyang, China
}

\begin{abstract}
As the demand increases, future networks will follow the trends of network variety and service flexibility, which requires heterogeneous type of network deployment and reliable communication method. In practice, most communication failure happens due to the bad radio link quality, i.e., high-speed users suffers a lot on the problem of radio link failure, which causes the problem of communication interrupt and radio link recovery. To make the communication more reliable, especially for the high mobility users, we propose a novel communication handoff mechanism to reduce the occurrence of service interrupt. Based on computer simulation, we find that the reliability on the service is greatly improved.
\end{abstract}

\section{Introduction}

To satisfy the increasing demand on the service rate, multiple types of base stations (BSs) are considered in the future network deployment, which consists of heterogeneous networks. However, various BS coverage sizes cause unreliable communication link when users moves from one big BS to a small BS or vice versa [1-5].

To solve the above problem and reduce the service interrupt rate, we propose a reliability enhanced handoff mechanism, which avoids the high-speed users to be handed over to the small BS due to its possible short stay, and muting the small BS signal transmission for some time to alleviate the interference when the high-speed use passing by. In this way, communication overhead can be greatly saved due to the avoidance on the unnecessary handoff and radio link recovery process.

The remaining part of this paper is organized as follows. In section 2, we formulate handoff problem for high-speed users in heterogeneous networks. The proposed enhanced handoff method is presented in section 3. The proposed method is evaluated in section 4 . Conclusions are drawn in section 5.

\section{Problem Formulation}

In this paper, we consider the small BSs are deployed in the coverage of big BS for the service rate enhancement as in Figure1, where users may suffer the interference from both big and small BSs.

Radio link failure is detected by BS based on the user's physical channel signal transmission. If BS finds that the user's signal transmission is in out-of-synchronization state and does not recover within some fixed time interval, then BS will think the radio link is suffering some problems and all the signals from the user will be neglected. After that, a process called radio link recovery will be initiated to make the user's signal transmission be back to the in-synchronization state. Usually, the synchronization state is based on the received signal-tonoise and interference ratio (SINR) of the synchronization preamble or pilot, and the value SINR should be smoothed as the filter in (1).

$$
F_{n}=(1-\alpha) \cdot F_{n-1}+\alpha \cdot M_{n}
$$

where $M_{n}$ is the simultaneous received SINR; $F_{n}$ is the updated filtered SINR, which is used to decide the user's synchronization state and link quality; $F_{n-l}$ is the previous SINR, where $F_{0}$ is set to $M_{l}$ when the first SINR is obtained; and $a=1 / 2^{(\mathrm{k} / 4)}$ is typically used with $k$ is the filter coefficient [6]-[7].

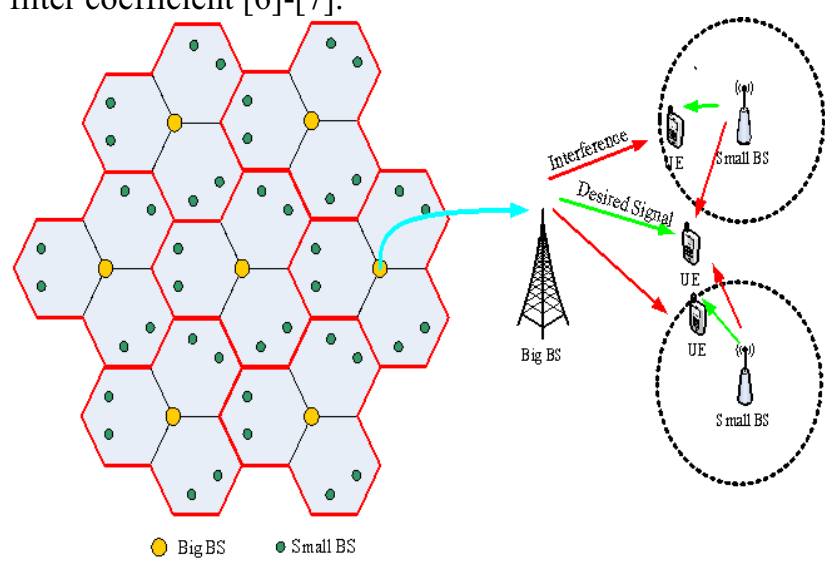

Figure 1. Illustration of heterogeneous network deployment considered. 


\section{Proposed Reliability Enhanced Handoff Method}

\subsection{Muted Subframe}

To relieve the interference from other BSs when highspeed users passing the small BS, some subframes of small BS are muted, which are also known as almost blank subframes (ABS). In these muted subframes, there is little signal transmission except the necessary control signals. In this way, the interference limited user is protected from the strong interference. But the subframe utility is a little reduced by the existence of muted subframes. To achieve a better trade-off, the muted subframe configuration should be optimized, i.e., the density and pattern are the key factors of performance.

\subsection{Proposed Reliability Enhanced Handoff Method}

To avoid the unnecessary handoff of high-speed user into the small BS's coverage and protect them from interference when passing by the small BS, additional steps such as user speed classification and muted subframe related configuration are introduced into the existing handoff procedure as in [8].

Here, we take a typical scenario as an example to illustrate the implementation of our proposed idea for a better understanding. The detailed procedure of the proposed algorithm can be found in Fig. 2. The proposed additional steps are as follows:

\section{Step-Measurement Reports with High-speed USER Indicator}

When the high-speed user gets closer to the small BS, an event called A3 may be triggered due to the stronger receiving power from the small $\mathrm{BS}$ than the serving big BS. In addition to the conventional measurement report, the high-speed user may also send an additional highspeed user indictor (i.e., one bit information with value ' 1 ' indicating high-speed user and valuser' 0 ' indicating other users) to the big BS to notify that the handoff to small BS should be avoided.

The user's mobility state estimation can be made by counting the number of handoffs within a specific time as usual or using the user handoff history information exchanged between BSs.

\section{Step-Small BS Muted Subframe Turn-on Request}

If the user is in high-speed state, big BS sends the "Muted Subframe Turn-on Request" message to the target small BS. When the big BS is providing VoIP service to the high-speed user now, the muted subframe turn-on Request also includes the next transmission timing information of the VoIP service.

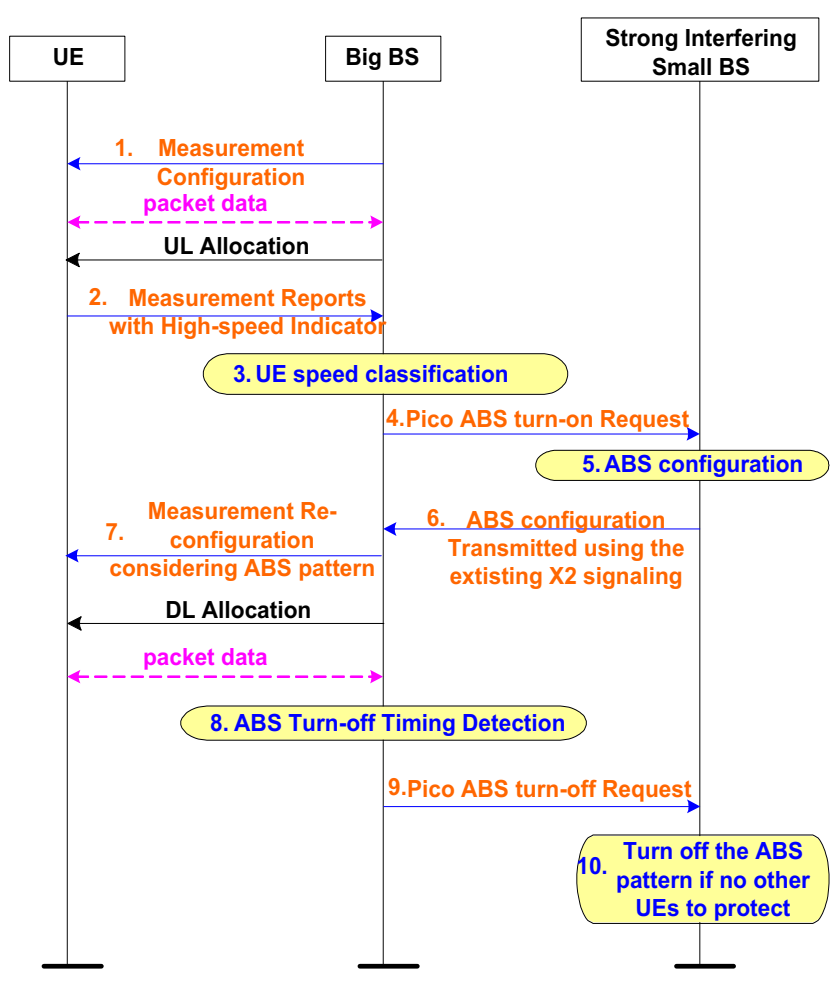

Figure 2 Detailed procedure for the proposed reliability enhanced handoff method

\section{Step-Small BS Muted Subframe Configuration}

When the small BS receives the "Muted Subframe Turn-on Request" from the big BS, some frames will be muted to protect high-speed user, but the muted subframe density configuration method is out of the scope of this paper. According to the small BS's current muted subframe status, the following actions can be taken by small BS.

a) If there is no muted subframe available at small BS now and the high-speed user is served with VOIP, then small BS will configure the muted subframe considering the high-speed user's next transmission timing information in the "Muted Subframe Turn-on Request" and VoIP data transmission interval to decide which subframe to be muted.

b) If there is no muted subframe at small BS now and the high-speed user is not requiring VoIP service, then Small BS will configure any subframe as the muted subsubframe.

c) If the small BS has already configured the muted subframe and the incoming "Muted Subframe Turn-on Request" is not for the VoIP user, no additional configuration is required.

d) If the small BS already configured the muted subframe and the incoming "Muted Subframe Turn-on Request" is for the VoIP user, small BS should reconfigure the muted subframe considering the next transmission information in the "Muted Subframe Turnon Request". 


\section{Step-Small BS Muted Subframe Configuration Exchange}

After small BS has configured or re-configured the muted subframe for the high-speed user, it will transmit the configuration information to the corresponding big BSs which have high-speed users to be protected by the muted subframe at this small BS, using the current existing interface between BSs.

\section{Step- High-speed USER RRC Reconfiguration}

After receiving the muted subframe configuration from small BS, the big BS will reset the measurement configuration through RRC signals and make the scheduling decisions considering the small BS muted subframe configuration.

\section{Step-Muted Subframe Turn-off Timing Detection}

The muted subframe adopted at small BS is used to protect the high-speed user, therefore the muted subframe should be turned off to avoid the resource waste when the high-speed user leaves the small BS's coverage.

There are two ways to detect the timing to turn-off muted subframe at small BS:

a) Explicit Detection

When the high-speed user detects the received RSRP or RSRQ from small BS below some predefined threshold, then it will send some specific message to its serving big $\mathrm{BS}$ to indicate the interference from the small BS is not serious now and the muted subframe at small BS can be turned off now.

b) Implicit Detection

When the serving Big BS receives another event A3 triggered measurement report with target BS ID is not the same small BS as that in the previous event A3 triggered measurement report from the high-speed user, the serving big BS knows that the high-speed user has already left the coverage of previous small BS and the muted subframe at that small BS can be turned off.

\section{Step-Small BS Muted Subframe Turn Off}

When big BS thinks there is no need to keep the muted subframe on for its serving high-speed user, it will send the "Muted Subframe Turn-off Request" to the corresponding small BS. After receiving this request, the small BS will decide whether to turn off the muted subframe or not.

\section{Evaluation Results}

\subsection{Simulation Parameters}

The detailed simulation parameters are outlined in Table 1 as same as that in [9]. Users are associated to a big or a small BS according to its best downlink reference signal receiving power. In addition to the data transmission, HARQ re-transmission is also considered in the performance evaluation.
Table 1 System simulation parameters

\begin{tabular}{|c|c|}
\hline Parameter & Value \\
\hline $\begin{array}{l}\text { HTN } \\
\text { scenario }\end{array}$ & $\begin{array}{l}\text { 3GPP downlink, outdoor Pico/Hotzone, } \\
\text { configuration 1, model } 1\end{array}$ \\
\hline $\begin{array}{l}\text { Cellular } \\
\text { layout }\end{array}$ & $\begin{array}{l}\text { Hexagonal layout with wrap around, } 7 \\
\text { eNodeBs, } 3 \text { cells per eNodeB }\end{array}$ \\
\hline $\begin{array}{l}\text { System } \\
\text { frequency }\end{array}$ & 2GHz carrier, $10 \mathrm{MHz}$ bandwidth \\
\hline ISD & $500 \mathrm{~m}$ (case 1$)$ \\
\hline $\begin{array}{l}\text { Max MeNB } \\
\text { Tx power }\end{array}$ & $46 \mathrm{dBm}$ \\
\hline $\begin{array}{l}\text { Max Pico Tx } \\
\text { power }\end{array}$ & $30 \mathrm{dBm}$ \\
\hline $\begin{array}{l}\text { Number of } \\
\text { Picos per cell }\end{array}$ & 2 , fixed at cell edge \\
\hline $\begin{array}{l}\text { Number of } \\
\text { UE per cell }\end{array}$ & 25 , uniformly and randomly distributed \\
\hline Traffic model & Full buffer \\
\hline $\begin{array}{l}\text { Scheduling } \\
\text { algorithm }\end{array}$ & Round Robin \\
\hline $\begin{array}{l}\text { Scheduling } \\
\text { delay }\end{array}$ & $6 \mathrm{~ms}$ \\
\hline $\begin{array}{l}\text { Scheduling } \\
\text { granularity }\end{array}$ & 5PRBs \\
\hline $\begin{array}{l}\text { Downlink } \\
\text { HARQ }\end{array}$ & $\begin{array}{l}\text { Asynchronous HARQ with CC, Maximum } \\
\text { three retransmissions }\end{array}$ \\
\hline $\begin{array}{l}\text { Channel } \\
\text { model }\end{array}$ & $\begin{array}{l}\text { SCM urban macro high spread for 3GPP } \\
\text { case } 1\end{array}$ \\
\hline $\begin{array}{l}\text { Number of } \\
\text { MeNB } \\
\text { antenna }\end{array}$ & 1 Tx antenna \\
\hline $\begin{array}{l}\text { Number of } \\
\text { Pico/Hotzone } \\
\text { antenna }\end{array}$ & 1 Tx antenna \\
\hline $\begin{array}{l}\text { Number of } \\
\text { UE antenna }\end{array}$ & $2 \mathrm{Rx}$ antennas \\
\hline $\begin{array}{l}\text { Downlink } \\
\text { receiver type }\end{array}$ & MRC \\
\hline $\begin{array}{l}\text { Channel } \\
\text { estimation } \\
\text { error }\end{array}$ & None \\
\hline $\begin{array}{l}\text { Pico Bias } \\
\text { Configuration } \\
\text { for Cell } \\
\text { Extension }\end{array}$ & $0,6,9 \mathrm{~dB}$ \\
\hline $\begin{array}{l}\text { UE Mobility } \\
\text { Model }\end{array}$ & Bouncing Circle \\
\hline $\begin{array}{l}\text { High } \\
\text { Mobility } \\
\text { Classification }\end{array}$ & $>60 \mathrm{~km} / \mathrm{h}$ \\
\hline Running time & $60 \mathrm{~s}$ \\
\hline
\end{tabular}

\subsection{Simulation Results}

In this section, we present the benefits of the proposed method in the aspects of the system's throughput and radio link failure rate. To simplify the analysis and make a fair comparison, we assume the handoff is perfect without any failure. To avoid ping-pong and the short time of stay problem, the high speed user is not allowed to be hand off to the small BS for any cases. 


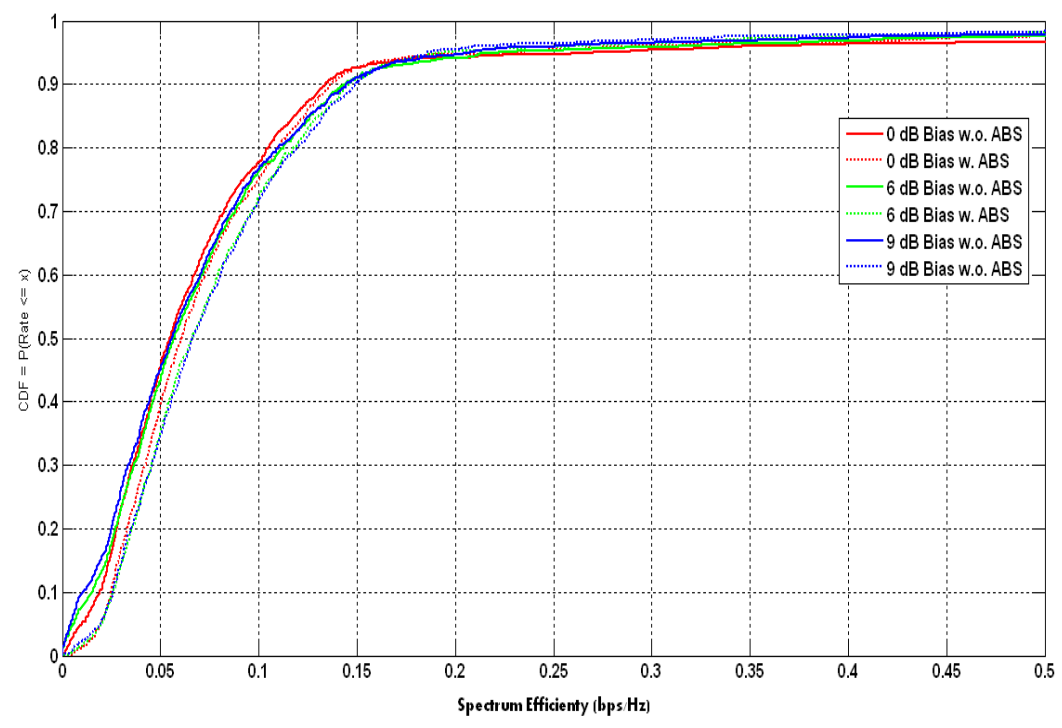

Figure 3. CDF of user spectrum efficiency in the cases of with and without muted subframe (ABS).

From the simulation results in Table 2, we find that the small BS's interference induced radio link failure is not negligible for the high-speed user if we avoid the highspeed user being handed off to the small BS even with OdB bias for small BS's coverage extension. We also find that the small BS muted subframe (a.k.a ABS) induced gain on RLF reduction is over $60 \%$, and in the case of large bias configuration such as $6 \mathrm{~dB}$ and $9 \mathrm{~dB}$, both the high-speed and low-speed users enjoy this gain.

Figure 3 shows the UE spectrum efficiency comparison between the cases of with and without ABS at small BS in the aspects of cumulative distribution function (CDF). A detailed comparison is shown in Table 3. From the results in Table 3, we find the ABS at small cell increases the user's received SINR, which covers the system resource loss due to ABS at small BS.

\section{Conclusions}

In this paper, we propose a reliability enhanced handoff method, which modifies the existing handoff procedure by adding some mobility related steps for system performance improvement. From the results, we observe that the performance of both radio link failure rate and system efficiency is greatly improved. For some system configuration such as high small BS bias case, even low speed users will benefit from the proposed method.

Table 2. RLF Rate with different small BS bias configuration

\begin{tabular}{|l|c|c|c|c|}
\hline $\begin{array}{c}\text { Small } \\
\text { BS Bias }\end{array}$ & $\begin{array}{c}\text { Speed } \\
(\mathbf{k m} / \mathbf{h})\end{array}$ & $\begin{array}{c}\text { W.o. ABS } \\
\text { (RLFs/UE/S) }\end{array}$ & $\begin{array}{c}\text { w. ABS } \\
\text { (RLFs/UE/S) }\end{array}$ & $\begin{array}{c}\text { Gain on RLF } \\
\text { Reduction }\end{array}$ \\
\hline \multirow{2}{*}{$\mathbf{0 ~ d B}$} & $\mathbf{3 0}$ & 0 & 0 & 0 \\
\hline \multirow{2}{*}{$\mathbf{6} \mathbf{~ d B}$} & $\mathbf{1 2 0}$ & 0.030 & 0.006 & $78.46 \%$ \\
\hline \multirow{2}{*}{$\mathbf{9} \mathbf{~ d B}$} & $\mathbf{3 0}$ & 0.010 & 0.001 & $86.76 \%$ \\
\cline { 2 - 5 } & $\mathbf{1 2 0}$ & 0.056 & 0.018 & $66.48 \%$ \\
\cline { 2 - 5 } & $\mathbf{3 0}$ & 0.046 & 0.008 & $81.63 \%$ \\
\hline
\end{tabular}

Table 3. Spectrum efficiency comparison between the cases of with and without muted subframe (ABS) at small BS

\begin{tabular}{|c|c|c|c|c|c|c|}
\hline $\begin{array}{l}\text { Small } \\
\text { BS } \\
\text { Bias }\end{array}$ & $\begin{array}{l}\text { Average } \\
\text { SE for } \\
\text { all UEs } \\
\text { w.o. } \\
\text { ABS } \\
\text { (bps/Hz) }\end{array}$ & $\begin{array}{l}\text { Average } \\
\text { SE for } \\
\text { all UEs } \\
\text { w. ABS } \\
\text { (bps/Hz) }\end{array}$ & $\begin{array}{l}\text { ABS } \\
\text { gain on } \\
\text { Average } \\
\text { SE for } \\
\text { ALL } \\
\text { UEs }\end{array}$ & $\begin{array}{l}\text { Average } \\
\text { SE for } \\
\text { High- } \\
\text { speed } \\
\text { UEs } \\
\text { w.o. } \\
\text { ABS } \\
\text { (bps/Hz) }\end{array}$ & $\begin{array}{l}\text { Average } \\
\text { SE for } \\
\text { High- } \\
\text { speed } \\
\text { UEs w. } \\
\text { ABS } \\
\text { (bps/Hz) }\end{array}$ & $\begin{array}{l}\text { ABS } \\
\text { gain on } \\
\text { Average } \\
\text { SE for } \\
\text { high } \\
\text { speed } \\
\text { UEs }\end{array}$ \\
\hline $0 \mathrm{~dB}$ & 0.0905 & 0.0925 & $2.21 \%$ & 0.0486 & 0.0585 & $20 \%$ \\
\hline $6 \mathrm{~dB}$ & 0.0887 & 0.0933 & $5.19 \%$ & 0.0484 & 0.0611 & $26.24 \%$ \\
\hline $9 \mathrm{~dB}$ & 0.0804 & 0.0893 & $11.07 \%$ & 0.0472 & 0.0623 & $31.99 \%$ \\
\hline
\end{tabular}

\section{Acknowledgement}

This research is supported by the research grant (with No. of L2014067) of Liaoning Provincial Education Department of China.

\section{References}

1. A. Racz, A. Te,evary, and N. Reider, "Handover performance in 3GPP long term evolution (LTE) systems," in Proc. MWCS, 2007, pp. 1-5.

2. A. Ulvan, R. Bestak, and M. Ulvan, "The study of handover procedure in LTE-based femtocell network," in Proc. IEEE WMNC, 2010, pp. 1-6.

3. D. Aziz, and R. Sigle, "Improvement of LTE handover performance through interference coordination," in Proc. of IEEE Veh. Tech. Conf. (VTC-spring), 2009, pp. 1-5.

4. K. Kitagawa, T. Komine, T. Yamamoto, and S. Konishi, "Performance evaluation of handover in LTE-Advanced systems with pico cell range 
extension,” in Proc. IEEE PIMRC, 2012, pp. 10711076.

5. R2-120652, On UE-speed-based methods for improving the mobility performance in HetNets, ALU, Feb. 2012.

6. Evolved Universal Terrestrial Radio Access (EUTRA); Mobility enhancements in heterogeneous networks, 3GPP TS 36.839, V11.1.0, Jan. 2013.

7. Evolved Universal Terrestrial Radio Access (EUTRA); Radio resource control (RRC); protocol specification, 3GPP TS 36.331, V11.3.0, Mar. 2013.

8. Evolved Universal Terrestrial Radio Access (EUTRA) and Evolved Universal Terrestrial Radio Access Network (E-UTRAN); Overall description; Stage 2, 3GPP TS 36.300, V11.5.0, Mar 2013.

9. Evolved Universal Terrestrial Radio Access (EUTRA); Further advancements for E-UTRA physical layer aspects, 3GPP TS 36.814, V9.0.0, Mar. 2010 